\title{
Diarrea Refractaria
}

Dr. Francisco Barrera Q.1; Dr. Dino Curotto A. 2 ; Dr. Pablo Avendaño U.3;

Dra. América Espinoza N. ${ }^{3}$; Nutr. Gladys Romero H.4 ; Enf. Sonja Escobar M.5

\section{Protracted Diarrhea}

Fifteen infants hospitalized because of protracted diarrhea between 1981 and 1984 were managed with dietetic treatment wich included Modular Diet, thus avoiding the use of total Parenteral Nutrition. Eventually all them rccovered. Their clinical presentation and course are analyzed.

Algunos casos de diarrea en el lactante evolu. cionan con un curso prolongado que puede llegar a hacerse refractario a los esquemas terapéuticos disponibles. En estos pacientes existen complejas alteraciones histológicas, funcionales y bioquímicas, muchas veces es imposible demostrar una etiologia, siendo frecuentemente necesario emplear procedimientos de alimentación de excepción y otras medidas terapéuticas de uso restringido.

1 Médico Unidad Lactantes B. Servicio de Pediatría Hospital Paula Jaraquemada. Docente Departamento de Pediatria Lniversidad de Chile.

2 Jefe Unidad Lactantes B. Docente Departamento de Pediatría Universidad de Chule.

${ }^{3}$ Becados del Servicio de Pediatría Hospital Paula Jaraquemada.

4 Nutricionista Servicio de Pediatría. Hospital Paula Jaraquemada.

5 Enfermera Lnidad Lactantes B. Serricio de Pediatría Hospital Paula Jaraquemada.

Trabajo ingreso Sociedad Chilena de Pediatría.
A continuación se presenta la experiencia recogida en el manejo de 15 casos de diarrea refractaria, analizando algunos aspectos clínicos, de laboratorio y tratamiento.

\section{MATERIAL Y METODO}

Durante el periodo comprendido entre el $1^{0}$ de Mayo 1981 y el 30 de Abril de 1984, 15 lactantes menores hospitalizados por diarrea refractaria fueron derivados a la Unidad de Lactantes B del Servicio de Pediatría del Hospital Paula Jaraquemada. Dos pacientes provenían de otros hospitales de Santiago y Valdivia y 13 de las otras unidades del Hospital. El criterio de diarrea refractaria corresponde al de Greene y cols. ${ }^{4}$, que se refiere a las diarreas que duran más de 4 semanas, se acompañan de compromiso en la ganancia de peso, y no tienen etiología demostrada.

Todos los pacientes fueron tratados con Dieta Modular (D.M.), que consistió en la administra- 
ción oral, programada, secuencial y progresiva, de los distintos módulos de nutrientes en sus formas menos complejas, utilizando came de res como fuente de proteinas, glucosa, fructosa, aceite vegetal y triglicéridos de cadena mediana (M.C.T.), completados con fleboclisis de soluciones de glucosa, electrolitos y vitaminas ${ }^{1+12}$, cuyo esquema se analiza en forma detallada en otra conunicación de este número 17.

El manejo de Ja diarrea refractaria incluyó siempre Nistatina oral u otro antimicótico, antibióticos de amplio espectro, plasma o transfusión de sangre total y ocasionalmente cubrió algunas carencias especificas tales como fósforo y zinc.

\section{RESULTADOS}

De los 15 lactantes 10 tenían menos de 5 meses y 12 presentaban, al comenzar la D.M., desnutrición de $2^{\circ}$ o $3^{\circ}$ grado según el criterio peso para la edad de M. Sempé.

Sólo tres pacientes habian recibido alimentación natural antes del ingreso. Todos los demás habiar sido alimentados artificialmente desde recién nacidos. La duración total de la diarrea hasta que se inició la D.M. varió entre 16 y 82 dias, con un promedio de 42 dias. El período de hospitalización varió de 29 a 160 , con un prome- dio de 66 días, una lactante enviada de provincia para nutrición parenteral total tuvo la diarrea más larga y la mayor estadía hospitalaria. Todos los pacientes habian recibido antes del comienzo de la D.M. múltiples antibióticos de "amplio espectro" por períodos prolongados, y también dietus restringidas, en última instancia leche sin lactosa (A1 110) o derivados de soya (Prosobee).

Once de los 15 pacientes tuvieron en algún momento de su evolución, antes de la introducción de D.M., uno o más coprocultivos positivos para algún enteropatógeno (en 3 casos Salmonella Tiphimurium, en 7 Echerichia coli enteropatógeno de serotipos clásicos y en 1 caso Klebsie1la). Un lactante sufrió una septicemia por Cándida albicans que fue tratada con Ketoconazol (R) oral y Amfotericina B parenteral. Once lactantes presentaron, en algún momento de su evolución, síndrome poliúrico funcional. En ocho se constataron concentraciones bajas de urea en la sangre, compatibles con síndrome de depleción de Urea. La poliuria cedió en todos coincidiendo con la estabilización de las concentraciones de $\mathbf{N H}_{3}$ plasmático y la proteinemia, excepto en dos casos, considerados como refractariedad secundaria a la hormona antidiurética, en quienes se observó una lenta recuperación de tos volúmenes urinarios normales (ver Tabla 1).

Tabla 1.

Características generales de lactantes que llegaron a dieta modular

\begin{tabular}{|c|c|c|c|c|c|c|c|c|c|c|c|c|c|c|c|}
\hline t ason blitice & 1 & 2 & 3 & + & 5 & 6 & $?$ & $B$ & 9 & in & u & 12 & 13 & 14 & 15 \\
\hline I. dat tmexest & 012 & 011 & a11 & 013 & Dt8 & 319 & $01 \leq$ & 014 & 013 & $0.1=$ & $\mathrm{NH}$ & 114 & $f \circ$ & 199 & $t: \leq$ \\
\hline Stxo & M & F- & no & 1. & 1 & N & M & r & M & : & 1: & M & y & м & t. \\
\hline 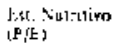 & b3 & $\mathrm{t}$ & $2 y$ & $123^{\circ}$ & D3 3 & L3 & 13 & 1 & vì & D) 2 & ma & DI & I. & 138 & 1J? \\
\hline Alumed: retho & No & $s_{i}$ & No & Nii & so & No & vis & No & vit & No & No & $\mathrm{si}$ & $N_{i}$ & No & $s_{1}$ \\
\hline $\begin{array}{l}\text { Duracicn } \\
\text { dratrea }\end{array}$ & 43 & IT & 31 & 82 & 35 & 16 & 29 & $\cdot 36$ & so & 18 & 21 & 14 & 21 & 27 & 53 \\
\hline $\begin{array}{l}\text { Dia t.sppi. } \\
\text { altuáứn }\end{array}$ & 73 & 89 & 29 & 56 & 160 & 62 & 36 & $\eta$ & 34 & of: & hij & $4 \mathbf{u}^{2}$ & 37 & 70 & $12 \pi$ \\
\hline $\begin{array}{l}\text { Altchent. } \\
\text { previos a D.M }\end{array}$ & AL 110 & AL, 110 & AL 110 & AL 113 & Putia & AL $1: 0$ & AL 110 & AL 110 & AL $11 \dot{U}$ & AL 110 & 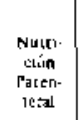 & $\begin{array}{c}\text { Prows. } \\
\text { bee }\end{array}$ & $A L \cdot 1 ?$ & 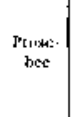 & AL 110 \\
\hline $\begin{array}{l}\text { Diufiss } \\
\text { mol:kg dia }\end{array}$ & 14: & 104 & 39 & 152 & 14 & $: 49$ & 119 & 82 & $1+0$ & & 83 & 24 & 120 & $1 ; 4$ & 05 \\
\hline 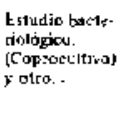 & $\begin{array}{l}\text { S.ti- } \\
\text { phim }\end{array}$ & $\begin{array}{l}\text { S. it. } \\
\text { plua: }\end{array}$ & is & $\begin{array}{c}\text { E.. Cali } \\
0.26\end{array}$ & 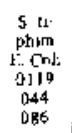 & 1) & $\begin{array}{c}\text { Uraculat. } \\
\text { Klebyellas }\end{array}$ & $\begin{array}{l}\text { L. Coli } \\
0127\end{array}$ & $\underset{\substack{\text { Coss } \\
\text { C. Col }}}{2}$ & $(1)$ & $\begin{array}{c}1, \cos \\
\text { 1,20 } \\
\text { andidas } \\
\text { Altricans }\end{array}$ & 1. culi & $\begin{array}{c}\text { 1: colt } \\
\text { DetT }\end{array}$ & 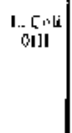 & 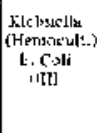 \\
\hline
\end{tabular}

La clasificación del Esstado Nucricional corresponde a Pe\$o/edad según M. Sempè.

La duración de la Diarrea y los días de hospitalización se tefieten al tiempo transcurrido hasta el inicio de la D.M. Los casos 3 y 5 fueron derivados de otros centros hospitalarios.

AL 110: fómula comercial que contiene Leche sin lactosa (sólo glucosa como hiạrato de carbono). 
La Fig. 1 muestra el importante compromiso del estado nutritivo de uno de los lactantes, que se expresaba en baja de peso, disninución crítica de los niveles de proteína y albúmina sérica, situáción que mejoró con el adecuado manejo de la diartea refractatia y el uso de D.M. En $87 \%$ de los lactantes se observó grados variables de disminución de proteinemia y albuminemia. Del mismo modo y en relación con el insuficiente aporte de proteínas y energía, se observó en $53 \%$ de los nir̃os descenso de las concentraciones de $\mathrm{NH}_{3}$ que los llevó a síndrome poliúrico.

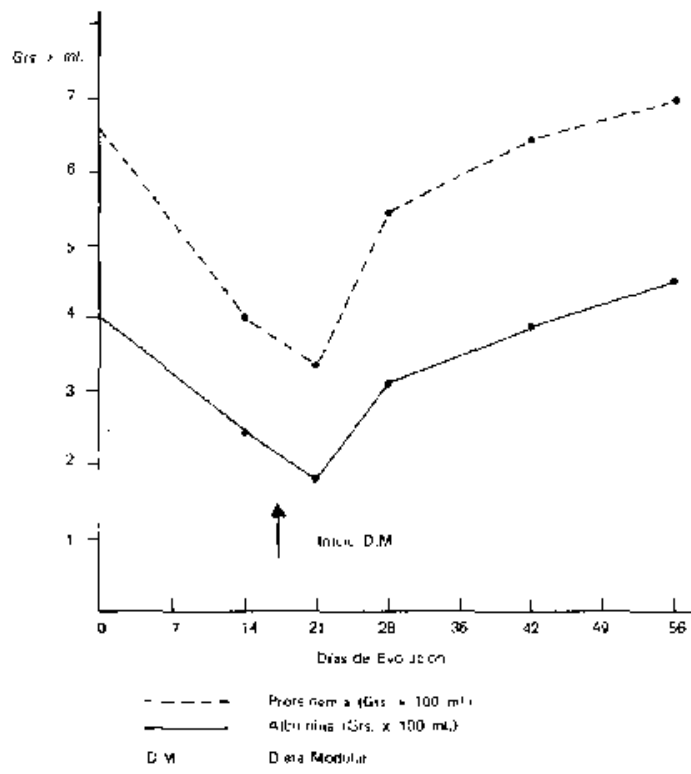

Figura 1. Dieta refractaria. Concentración de proteinas y albúmitia en el sucro de un lactante con diarrea refractaria.

La Tabla 2, muestra la mejoría asociada con el empleo de D.M. en uno de los lactantes. A menudo se observó ausencia de deposiciones en las sigujentes 24 a 48 horas después del comienzo de la D.M. lo que podría explicarse por el control ejercido por la D.M. sin glucosa, administrada en los primeros tres días de tratamiento, ante una disminución crítica de la tolerancia intestinal por la glucosa. Posteriormente tanto en el paciente analizado como en los demás, el volumen de deposiciones se mantuvo entre 5 y $15 \mathrm{cc} \times \mathrm{k}$. de peso por día.

Tabla 2.

Modificaciones del balance metabólico de un paciente sometido a dieta modular

Caso 6 D.A.L. \#67281

Balance nuctabólico Previo DM 20 dia DM Térnino DM

Peso ing ceso (M (grs)

Peso egreso CM (grs)

$\begin{array}{rrr}5430 & 5100 & 5100 \\ 5380 & 5080 & 4956 \\ -50 & -20 & -144 \\ 61 & 172 & 119 \\ 1001 & 1010 & 1000 \\ 162 & 0 & 7.8 \\ 75 & 25 & 22\end{array}$

Diuresis (ce $x$ (

Densidad

Deposiciones (c $\times \mathrm{kg}$ ).

Pérdida inensibles

(cc $\mathrm{x} \mathrm{kg}$ ).

No

No

No

Natrerial Natrimia

(m) I.j/lt.)

$137 / 7 \quad 130 / 19 \quad 128 / 66$

Proporción orisal

plassua Na

0.05

0.15

0.5

Kalema/Kaliuria

unEq́lt.

Proporción orina/

plasma. $\mathrm{K}$.

$\mathrm{NH}_{3}$ plastisa

me\% $\%$

$3.5 / 11$

$2.4 / 3$

$4.1,18.5$

3. 14

1.25

4.4

9

11

Cuando hubo poliuria, ésta demoró más que la diarrea en desaparecer, lo que puede atribuirse en parte a la necesidad de aportar un gran volumen de agua debido al tipo de alimentos empleados y a la necesidad de mantener un suministro adecuado de proteinas y calorías. La Tabla 2 presenta además otros aspectos del balance metabólico onientados a evaluar la función renal.

La Tabla 3 muestra las variaciones de peso observadas durante la evolución de los pacientes. La desnutrición no se agravó con la D.M., observándose más bien mejoría en la redistribución del agua corporal, desaparición de edemas, mejor contacto con el medio ambiente, aunque sólo posteriormente comenzara un franco aumento

Tabla 3.

Modificacioneș en el peso de les lactantes sometidos a dieta modular (D.M.)

\begin{tabular}{|c|c|c|c|c|c|c|c|c|c|c|c|c|c|c|c|}
\hline Caso chimco & 1 & 2 & 3 & 4 & 5 & 6 & $?$ & 8 & $\dot{y}$ & 10 & $1 \mathbf{i}$ & 12 & 13 & 14 & Is \\
\hline Peso inlusesn (ycs.) & $33(-1)$ & 34000 & 6100 & 3450 & 4060 & $56 \mathrm{RO}$ & 5030 & 6300 & 2.770 & 2370 & 5820 & 6300 & 8310 & $\$ 450$ & $5 E 00$ \\
\hline Peso inicio DM (bूra) & 3250 & 3120 & 6100 & 3360 & 4000 & 5370 & 4920 & 6260 & 2350 & 4370 & 5600 & 5790 & 7940 & \$स्ष 10 & 4900 \\
\hline Pcso lénmino Uht (grs.) & 3150 & $3+21$ & 5980 & 3980 & 3640 & seros & 4820 & $6481 i$ & 2390 & 4330 & 6500 & 5670 & 7440 & 6000 & 5440 \\
\hline Duracion DM (días) & 20 & 18 & 18 & 14 & 30 & 24 & 12 & 42 & 10 & 12 & 20 & 12 & I? & 21 & 30 \\
\hline
\end{tabular}


ponderal. Algunos pacientes, seguidos ambulatoriamente, lograron posteriormente incrementos de peso de hasta 60 gramos diarios.

La D.M. se mantuvo por 10 a 24 días, cambiándose posteriormente, con excelente tolerancía, a derivados de Soya con Triglicéridos de cadena mediana (T.C.M.) (Prosobee más $2 \%$ de T.C.M.).

\section{DISCUSION Y COMENTARIO}

Nuestra experiencia concuerda con otras ${ }^{1-2-3}$, en que aproximadamente $1 \%$ de los pacientes hospitalizados por diarrea prolongada evolucionan de manera refractaria a los tratamientos habituales ${ }^{2-4}$. Se desconoce la razón, pero se cree que estaría relacionada con profundas modificaciones en la función intestinal, independientes muchas veces de la causa inicial y del tratamiento efectuado.

Se ha pretendido identificar a los niños con rjesgo de evolucionar hacia la refractariedad mediante distintos parámetros clínicos entre ellos edad, ausencia de lactancia natural, persistencia de bacterias enteropatógenas y estado nutritivo, sin embargo ninguno de ellos es confiable por sí solo. Tampoco existe una definición precisa del momento en que puede calificarse una diarrea como refractaria, pero la edad, la duración del trastorno, el compromiso de la nutrición y de la función intestinal son los aspectos más importantes, destacando la disminución de la tolerancia oral a los hidratos de carbono, incluyendo glucosa y fructosa $4 \cdot 10$.

No encontramos otras alteraciones en el equilibrio hidroelectrolítico y ácido base de estos niños, fuera del edema y los trastornos de distri. bución de los líquidos secundarios a la desnutrición pluricarencial, en lo que concidimos con otras publicaciones.

La aparición de un sindrome poliúrico funcional, habitualmente reversible, de patogenia compleja, sin relación con excesos de aporte de liquidos, tiene gran importancia clínica. Entre las posibles causas destacan las alteraciones renales del desnutrido que pueden tener origen en kaliopenia, expoliación de urea, refractariedad a la hormona antidiurética o nefritis intersticial perdedora de sodio (ocasionada por infección o tratamiento antibiótico). En nuestros casos se observó con frecuencia el síndrome de expoliación de urea que respondió adecuadamente una vez que el balance nitrogenado se hizo positivo.

Otro aspecto fundamental en la diarrea refractaria es el compromiso progresivo del estado nutritivo con desnutrición calórica inicial sobre la cual se pueden agregar grados variables de desnutrición protéica más o menos aguda, como con- secuencia del aumento de los requerimientos de energía y protejnas, ayunos reiterados, realimentaciones cautelosas con bajos aportes nutritivos que en general reciben los pacientes hospitalizados $^{6-6}$, círculo vicioso de enfermedad-desnutrición dificil de romper.

En algunos pacientes la infección que desencadenó el proceso puede seguir latente, en otros se producen sobreinfecciones con bacterias oportunistas y hongos, pudiendo ocurrir candidiásis superficiales y también sistémicas como en uno de nuestros pacientes. Finalmente también es posible que segmentos superiores del intestino sean colonizados por flora habitual de otros más bajos como ileón y colon provocando profundas alteraciones en los mecanismos de digestión y absorción ${ }^{10}$.

Los trastomos hemorrágicos observados ocasionalmente no han sido explicados satisfactoriamente.

El manejo adecuado de estos pacientes debe incluir la corrección inicial de trastornos hidroelectrolíticos y del equilibrio ácido-base existen. tes, dentro de las primeras $48 \mathrm{hrs}$. El empleo de técnicas de balance metabólico y de enfermería son fundamentales ${ }^{7}$. Se ha sugerido la administración inicial de plasnia como expandidor de volumen o apoyo en la enterocolitis asociada con inmunodeficiencias ${ }^{\theta}$.

A continuación debe realizarse una evaluación del tol de la infección bacteriana, o la coloniza. ción por flora saprófita de los segmentos superiores del intestino delgado. De ello dependerá la necesidad de indicar antibióticos apropiados. A este respecto consideramos conveniente usar siempre Nistatina oral y local (excepcionalmente otros antimicóticos sistémicos), pues existen varios factores predisponentes a las infecciones del sistema digestivo por Candida Albicans, aún con invasión sanguínea y localización distante (infección bacteriana, desnutrición, tratamiento antibiótico, deshidratación, punciones venosas, catéteres, fleboclisis prolongada) ${ }^{9-10}$. También puede ser conveniente usar Colestiramina (Que$\operatorname{tran} R$ ) o Colestipoid (ColestidR), resinas de amonio cuaternario que pueden beneficiar a un lactante con diarrea refractaria a través de la neutralización de ácidos biliares desconjugados, que suelen estar aumentados en el ileón teminal y colon, pữiendo causar persistencia de la diarrea a través de enteropatía colereica y un componente secretor ${ }^{13} \cdot 14-15-18$, Estas resinas además parecen útiles en la diarrea producida por exotoxinas de E. coli enteropatógena, cuyo efecto tóxico neutralizaria, ${ }^{19}$. Debe recordarse, sin embargo, que la Colesteramina está contraindicada cuando se sospecha obstrucción intestinal, peritonitis o hipoprotrombinemia, y que su uso 
en altas dosis por periodos prolongados produce esteatorrea y otras complicaciones secundarias. Usamos Colestipoid en 7 casos de esta serie en dosis de $0,5 \mathrm{~g} . \times \mathrm{k}$. de peso $\mathrm{x}$ día, se consideró contraindicada en 2 , por sospecha de peritonitis aguda en uno y esteatorrea moderada en otro. Creemos que su uso debe preceder al empleo de D.M. pues muchas veces ha sido eficaz en casos probables de enteropatía coleréica.

Finalmente debe hacerse siempre una prueba terapéutica con D.M., ya que la realimentación por via enteral en los pacientes con diarrea prolongada o refractaria acelera la recuperación bioquímica, histológica y funcional del intestino enfermo $4-5 \cdot 12-20$. En el caso de la diarrea refractaria, con insuficiencia intestinal que compromete el umbral de tolerancia a la glucosa, los módulos de nutrientes deben ser ofrecidos de manera secuencial, siguiendo un orden estricto relacionado con las caracteristicas de la recuperación de la función intestinal que se vaya obteniendo ${ }^{11.17}$.

\section{RESUMEN}

Se presentan 15 casos de diarrea refractaria, que requirieron tratamiento con Dieta Modular (D.M.), en la Unidad de Lactantes B del Servicio de Pediatıía del Hospital Paula Jaraquemada entre Jos años 1981 y 1984. Dos pacientes habian sido enviados de otros hospitales.

Los pacientes recibieron D.M. oral con introducción precoz de fructosa y triglicéridos de cadena mediana. Todos los casos evolucionaron favorablemente, observándose en una paciente una recaída debida a infección por salmonella typhimurium.

\section{REFERENCIAS}

1 Duffau G.: Síndrome diarréico del lactante. Santiago de Chile. Edit Andrés Bello, 1978.

2 Avery G.B., Villavicencio D., Lilly J.R. and Randotph $I . G .:$ Intractable diarthea in early infants. Pediatrics 41: 712, 1978.

${ }^{3}$ Sunshine P., Sinatra F.R., Mitchell C.H.: Intractable diarrhea of infancy, Clin. Gastroenterol. 6: 445, 1977.

4 Greene H.I., MC Cabe D.R., Merenstein G.D.:
Protracted diarthea in infancy, J. Pediat. 87: 695, 1975.

s Merrith R.I., Suskind R.M.: Nutritional survey of hospitalized pediatrics patients, Am. J. Clin, Nutr. 32: $1320,1979$.

6 Gattas V., Fuentes A., Jarpa S., Vouy R.: Situación alimentaria de pacientes pediátricos hospitalizados. Kev. Chil. Pediatr. 52: 397, 1981.

${ }^{7}$ Duffat G.: Metódica de balance en cama metabólica aplicada al estudio del sindrome diarréico agudo del lactante. Bol. Med. Infant. 34: 551, 1977.

${ }^{8}$ Cannon R.A., Blum P.M., Ament M.E., Byrne W.J., Soderberg Warner M., Seeger R.C.,Saxon A.E., Stiehm E.R.: Reversal of enterocolitis - associated combined immuno deficiency by plasma therapy, $\mathrm{J}$. Pediatr. 101: 711, 1982,

9 Mozunder P.K., Morks M.I.: Candida Albicańs infections in hospitalized children. A survey of predisponing factors, Clin. Ped. 14: 123, 1975.

10 Barnes G.L., Bishop R,R., Townley R.R.W.: Microbial flora and disaccharidasse depression in infantile gastroenteritis, Acta Paediatr. Scand. 63: $423,1974$.

11 Klish W.J., Potts E., Ferry G.D.: Modular Formula: and approach to management of infants with specific or complex food intolerances, J. Pediatr. 88: 948, 1976.

12 Lake A.M., Kleinmon R.R., Walker W.A.: Enteric alimentation in specialized gastrointestinal problems and alternative to total parenteral nutrition, Advances in Pediatrics 28: 319, 1981.

13 Heuby l.E. Balistreri W.F.: Bile salt metabolism in infants ard children after Protracted Infantile Diarrhea, Pediatr. Res. 14: 943, 1980.

14 Emilfork M., Duffau G., Bascur D.L., Urbina $A$.: Excreción fecal de ácidos biliares en el síndrome diarréico del lactante según forma evolutiva. Bol. Méd. Infant. 39:177, 1982.

15 Duffau G. Emilfork M., Bascur $I_{.,}$, Urbina A.M. Niveles séricos de ácidos biliares según forma evolutiva. Bol. Méd. Infant, 39: 83, 1982.

16 Tomer M.A., Santora R.R., Sandberg D.H.: Cholestyramine Therapy for Intractable Diarrhea Pediatrics. 53: 217,1974

17 Barrera F., Curotto D., Avendafio P., Romero G., Escober S.: Dieta Modular, Rev. Chil. Pediatr. 55(5), 1984.

18 Duffot $G$, Emilfork $M .$, Martínez A.: Colestiramina en el tratamiento de la diarrea refractaria del lactante. Bol. Med. Infant. 37: 979, 1980.

19 Duffau G.. Emilfork M. Prenzel I., Urbina A.M., Martinez A.: Colestiramina en el tratamicnto de la diarrea aguda bacteriana del lactante. Bol. Med. Infant. 36: $735,1979$.

20 Brown K., Mac Lean W.: Nutritional Management of Acute Diarrhea: An Appraisal of the Alternatives, Pediatres. 73: 119, 1984. 